\title{
Evaluation of Segmentation Techniques Using Region Size and Boundary Information
}

\author{
D.P. Dogra ${ }^{1}$, A.K. Majumdar ${ }^{1}$, and S. Sural ${ }^{2}$ \\ 1 Department of Computer Sc. \& Engineering, Indian Institute of Technology, \\ Kharagpur, 721302, India \\ \{dpdogra, akmj\}@cse.iitgp.ernet.in \\ 2 School of Information Technology, Indian Institute of Technology, \\ Kharagpur, 721302, India \\ shamik@sit.iitgp.ernet.in
}

\begin{abstract}
Image segmentation quality evaluation is a key element when comparing segmentation algorithms. In computer vision, unsupervised segmentation algorithms, although of great interest, often suffer from lack of a well-defined measure to evaluate. This paper presents a novel idea for evaluating such algorithms. A measure is proposed to evaluate four well referred segmentation algorithms. The metric proposed in this work is composed of both size and boundary of segments. When compared with some of the existing techniques, it is found that the proposed scheme can approximate the segmentation error in a better way.
\end{abstract}

Keywords: Segmentation Evaluation, Area Matching Index, Boundary Matching Index, Combined Matching Index.

\section{Introduction}

Image segmentation is an important ingredient that is used in many image analysis and computer vision applications. In spite of focusing extensively in designing segmentation techniques, relatively less attention has been given in evaluating those algorithms. Thus, development of a suitable evaluation technique that can be used to compare efficacy of segmentation algorithms.

The existing evaluation methods can broadly be divided into two categories: analytical and empirical methods [17]. A review of analytical based measures can be found in [11] [15]. Empirical methods indirectly judge the segmentation algorithms by comparing them with gold standard segmentation of the images under consideration. Though, most of these evaluation methods are subjective or tied to specific applications [16, a number of unsupervised objective evaluation measures [1] 8] 9] 10, 12, 14 have received considerable attention. Features like, region boundary, region size, region uniformity, region contrast etc. are used in unsupervised objective evaluation. Furthermore, these techniques can be classified into two groups, i.e. region boundary and size based techniques. The former evaluates segmentation in terms of accuracy of region's shape, while the latter approach assesses the segmentation quality in terms of overlap size. 
The evaluation methodology developed by Martin et al. [9] is a classic example of size based technique. Recently, a new size based measure using Normalized Probabilistic Rand (NPR) index is proposed [14. Alternatively, the boundary based approach proposed by the authors of [10 has been used in many applications. Also, precision and recall based method proposed by the authors of [5] [6] are popular. A drawback of precision / recall based scheme is that, given a correspondence between two segmentations, it is possible to change the locations of the unmatched boundary almost arbitrarily and retain the same precision and recall score.

Thus, a measure that considers both size and boundary of regions can be of great use in defining segmentation accuracy. We propose a technique that combines a recently proposed region size based measure [1] with a new boundary based measure. Rest of the paper is organized as follows. A detail description of the proposed measure is described in section 2. Evaluation strategy and results are reported in Section 3 while we conclude in Section 4 with some discussions.

\section{Proposed Method of Evaluation}

LCE-GCE method introduced by Martin et al. 9, NPR method proposed by Unnikrishnan et al. [14 and Segmentation Covering (SC) 1] are size based measures whereas methods proposed by the authors of [5] [6] [10] are boundary based. We explain the limitations of these methods with the example shown in Fig[1. Let, $G=\left\{G_{1}, G_{2}, \ldots . G_{m}\right\}$ and $S=\left\{S_{1}, S_{2}, \ldots \ldots S_{n}\right\}$ be two sets of segmentations where $G_{i}$ and $S_{j}$ are $i^{\text {th }}$ and $j^{\text {th }}$ segments of gold and machine segmentations respectively. Fig. 1(d) shows that $G_{2}$ has maximum intersection with $S_{2}\left(\forall j, S_{j} \in S\right)$ and it is a subset of $S_{2}$. These measures do not consider the common boundary information. NPR based method too does not incur

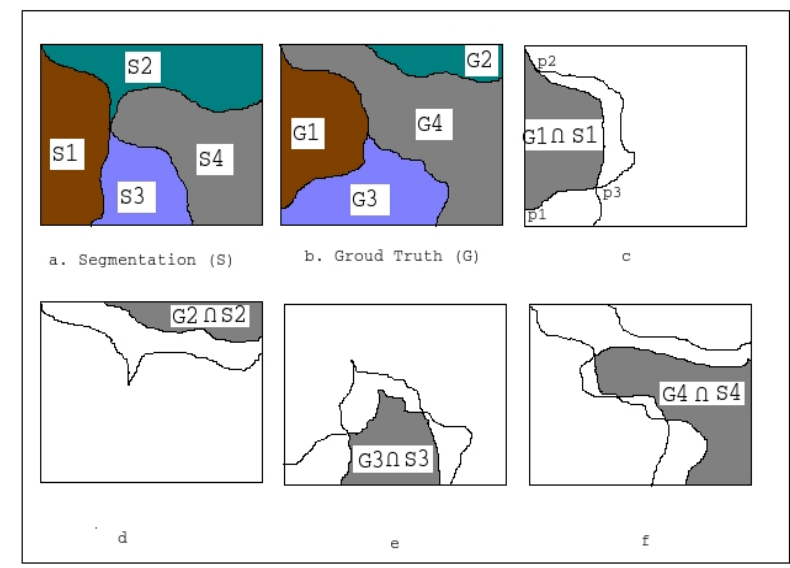

Fig. 1. (a) Computer Generated Segmentation Map of an Image. (b) It's Ground Truth Map. (c-f) Individual Intersection Map. 
additional penalty despite a high degree of dissimilarity between the boundaries. Thus, a measure that takes into account both size and boundary based errors, will be of great help in evaluating segmentation algorithms. To define the proposed measure, two separate indices are used, i.e. Area Matching Index (AMI) [1] and a new Boundary Matching Index (BMI). Out of the three size based measures discussed earlier, as SC [1] based approach is most recent and it performs reasonably well for natural images, we have adopted it in our work.

$$
A M I_{\text {overall }}=C(G \rightarrow S)=\frac{1}{N} \sum_{S_{j} \in S}\left|S_{j}\right| \cdot \max _{G_{i} \in G}\left\{O\left(S_{j}, G_{i}\right)\right\}
$$

where $S=\left\{S_{1}, S_{2}, \ldots \ldots, S_{n}\right\}$ and $G=\left\{G_{1}, G_{2}, \ldots \ldots, G_{m}\right\}$ and $\mathrm{N}$ is total number of pixels of the image and $O\left(S_{j}, G_{i}\right)$ is called the overlap between two regions $S_{j}$ and $G_{i}$. It is estimated by:

$$
O\left(S_{j}, G_{i}\right)=\frac{\left|S_{j} \cap G_{i}\right|}{\left|S_{j} \cup G_{i}\right|}
$$

A new boundary based measure that considers neighborhood information is proposed here. We call it as Boundary Matching Index (BMI). It is computed is as follows. Let, the closed arc $\mathrm{P}=\left\{p_{1}, p_{2}, p_{3}\right\}$ in Fig. 1(c) describes the boundary of $G_{1} \cap S_{1}$. Two situations may arise: (i) A point on the arc P, say p, is a common boundary point of both $G_{1}$ and $S_{1}$ (e.g. $x \in\left\{p_{1}, p_{2}\right\}$ ) (ii) The point on $\mathrm{P}$ is either on boundary of $G_{1}$ or $S_{1}$ (e.g. $x \in\left\{p_{1}, p_{3}\right\}$ or $\left.x \in\left\{p_{3}, p_{2}\right\}\right)$. A score $\left(B M I_{i j}\right)$ based on boundary points $(\mathrm{P})$ of the region $G_{1} \cap S_{1}$ is estimated by:

$$
B M I_{i j}=\frac{\sum_{p=1}^{k} C_{p}}{k} \quad \text { for } i=1 \text { and } j=1
$$

where $\mathrm{k}$ is the total number of points on $\mathrm{P}$ and $C_{p}$ is defined by:

$$
C_{p}=\left\{\begin{aligned}
1 & \text { If }\left|p-\operatorname{Boundary}\left(G_{i}\right)\right|<d \text { and }\left|p-\operatorname{Boundary}\left(S_{j}\right)\right|<d \\
\frac{N_{p}}{8}, \text { Otherwise } &
\end{aligned}\right.
$$

where $N_{p}$ is the number of neighboring pixels of p that belong to $G_{i} \cap S_{j}$ using 8 -connectivity rule and $d$ is a strictness measure normally set to 3 . A value of $d=1$ will over-penalize boundary points those are close but not coincident. Taking the average $B M I_{i j}$ of all machine generated segments, final score of the index as: $B M I_{\text {overall }}=\frac{B M I_{i j}}{n}$ for $i \in m$ and $j \in n$ is estimated. Finally, a Combined Matching Index (CMI) is produced by:

$$
C M I=\frac{W_{A M I} X A M I_{\text {overall }}+W_{B M I} X B M I_{\text {overall }}}{2}
$$

where $W_{A M I}$ and $W_{B M I}$ are weights for area and boundary index respectively and satisfy the condition $W_{A M I}+W_{B M I}=1$. 


\section{Evaluation Strategy and Results}

We choose four well referred segmentation techniques to evaluate the performance of the proposed measure namely, Mean Shift (MS) [2] 3], Normalized Cut (NC) [13, Efficient Graph Based Method (GB) 4] and Color Based Salient Region Segmentation (SR) [7]. Berkeley Segmentation Dataset (BSDS) 9] that contains 300 natural images including multiple manual segmentations, is used for evaluation of the proposed measure. Fig. 2 depicts the segmentations of an image taken from BSDS with five ground truth results. To represent the result shown in Fig. 2 in a better way, we have plotted the values of five measures including the proposed one using the graph shown in Fig. 3. It is understood from the graph that the proposed measure (CMI) rates the SR based segmentation algorithm with a significantly low matching index when compared to other measures. CMI performs equally well as compared to NPR for MS, NC and GB algorithms while a better approximation of segmentation error is evident particularly for SR algorithm.

We have applied four segmentation algorithms on the images of the BSDS dataset and recorded the values of five evaluation measures and found that the proposed measure does a better approximation of segmentation error. From Fig. 4, it is evident that the segmentation result produced by $\mathrm{NC}$, GB and MS are almost similar for most of the images and all measures including CMI agree in most of cases. Segmentation produced by SR algorithm for the second image is not satisfactory and CMI detects it with a high degree of confidence. Though all five measures evaluate the segmentation of the rock image (Fig. $4,3^{\text {rd }}$ row) in a consistent manner, CMI performs good approximation in case of bird image (Fig. 4, $1^{\text {st }}$ ) and old man image (Fig. 4, $2^{\text {nd }}$ ).

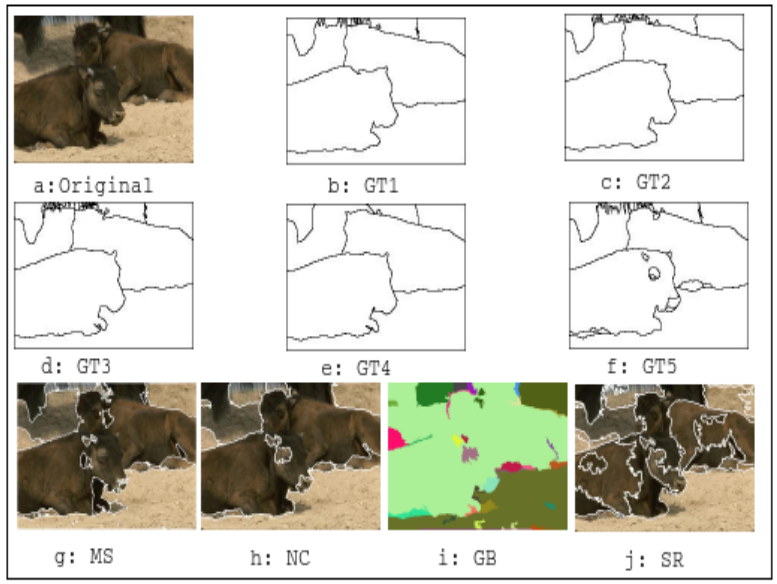

Fig. 2. Output of MS, NC, GB, SR Schemes with Five Ground Truth Segmentations 


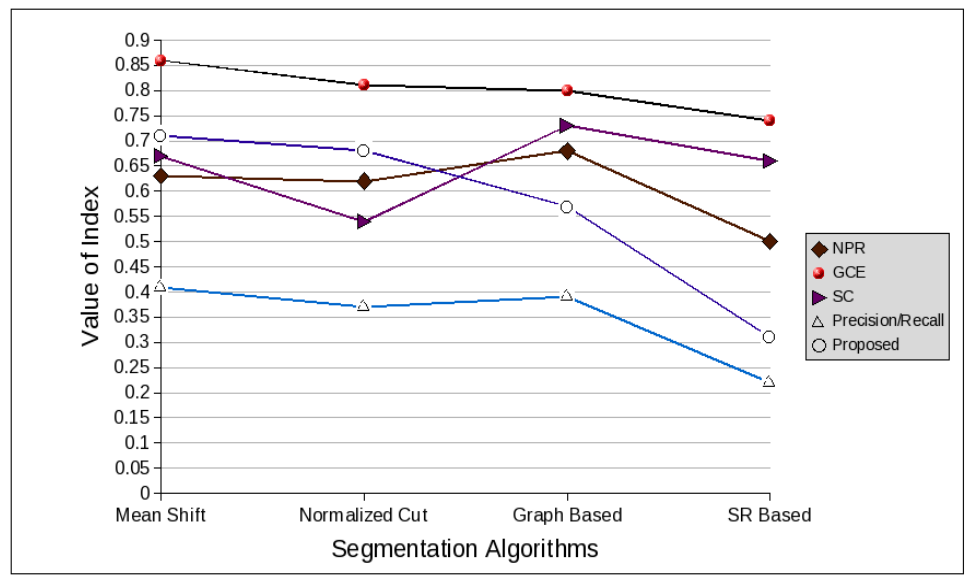

Fig. 3. Graphical Representation of Five Evaluation Measures for the Image of Fig. 2

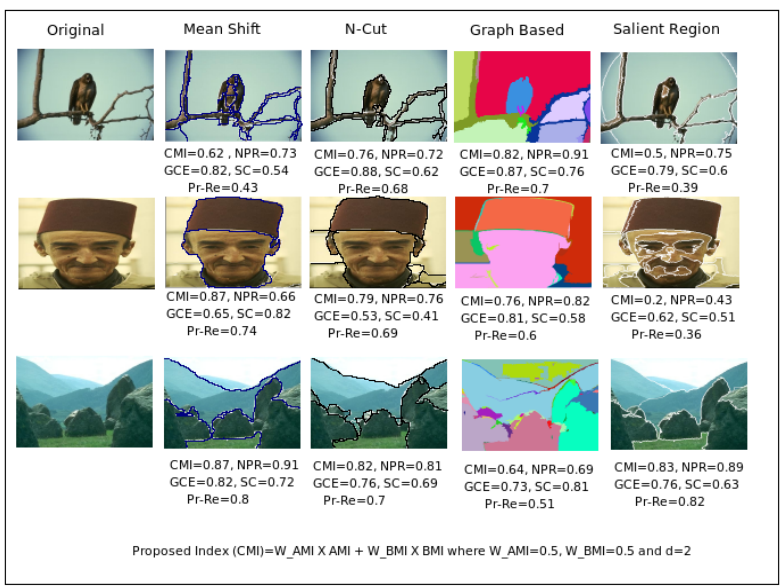

Fig. 4. Results using MS, NC, GB, SR Schemes and Recorded Evaluation Measures Applied on BSDS dataset

\section{Conclusion and Future Work}

Evaluation of segmentation schemes is an interesting problem in computer vision and image processing area. In this work, a new objective evaluation measure is proposed to compare segmentation algorithms. While most recent segmentation quality evaluation methods only deal with either of area or boundary, the proposed one uses both region size and boundary of the segmentation partition in a clean and comprehensive way. As a low level measure, it produces valid results under varying conditions where reference segmentation is available. This technique could also be used as a building block in more complex and application 
specific evaluation schemes. In conclusion, though efforts have been put on and encouraging results have been obtained in the last few years, new ideas and procedures for the evaluation methodology and their practical implementation are still required.

\section{References}

1. Arbelaez, P., Maire, M., Fowlkes, C., Malik, J.: From Contours to Regions: An Empirical Evaluation. In: CVPR (in press, 2009)

2. Comaniciu, D., Meer, P.: Mean Shift: A Robust Approach Toward Feature Space Analysis. IEEE Trans. on PAMI 24(5), 603-619 (2002)

3. Comaniciu, D., Meer, P.: Mean Shift Image Segmentation Software, http://www. caip.rutgers.edu/riul/research/code/EDISON/index.html

4. Felzenszwalb, D.: Efficient Graph-based Image Segmentation. IJCV 59(2), 167-181 (2004)

5. Freixenet, J., Muñoz, X., Raba, D., Martí, J., Cufí, X.: Yet Another Survey on Image Segmentation: Region and Boundary Information Integration. In: Heyden, A., Sparr, G., Nielsen, M., Johansen, P. (eds.) ECCV 2002. LNCS, vol. 2352, pp. 408-422. Springer, Heidelberg (2002)

6. Huang, Q., Dom, B.: Quantitative Methods of Evaluating Image Segmentation. In: ICIP, pp. 53-56 (1995)

7. Kuan, Y., Kuo, C., Yang, N.: Color-Based Image Salient Region Segmentation Using Novel Region Merging Strategy. IEEE Trans. on MM 10(5), 832-845 (2008)

8. Martin, D.: An Empirical Approach to Grouping and Segmentation. PhD Dissertation, Univ. of California, Berkeley (2002)

9. Martin, D., Fowlkes, C., Tal, D., Malik, J.: A Database of Human Segmented Natural Images and its Application to Evaluating Segmentation Algorithms and Measuring Ecological Statistics. ICCV 2, 416-425 (2001)

10. Martin, D., Fowlkes, C., Malik, J.: Learning to Detect Natural Image Boundaries Using Local Brightness, Color and Texture Cues. IEEE Trans. on PAMI 26(5), 530-549 (2004)

11. Pal, N.R., Pal, S.K.: A Review on Image Segmentation Techniques. Jour. of PR 26(9), 1277-1294 (1993)

12. Rand, W.: Objective Criteria for the Evaluation of Clustering Methods. Journal of ASA 66, 846-850 (1971)

13. Shi, J., Malik, J.: Normalized Cuts and Image Segmentation. IEEE Trans. on PAMI 22(8), 888-905 (2000)

14. Unnikrishnan, R., Pantofaru, C., Hebert, M.: Toward Objective Evaluation of Image Segmentation Algorithms. IEEE Trans. on PAMI 29(6), 929-944 (2007)

15. Weszka, J.S., Rosenfeld, A.: A.: Threshold Evaluation Techniques. IEEE Trans. on SMC 8(3), 622-629 (1978)

16. Zhang, H., Frittb, J.E., Goldman, S.A.: Image Segmentation Evaluation: A Survey of Unsupervised Methods. Jour. of CVIU 110(2), 260-280 (2008)

17. Zhang, Y.J.: A Survey on Evaluation Methods for Image Segmentation. Jour. of PR 29(8), 1335-1346 (1996) 